\title{
Control Oriented Prediction of Driver Brake Intention and Intensity Using a Composite Machine Learning Approach
}

\author{
Jianhao Zhou *, Jing Sun, Longqiang He, Yi Ding, Hanzhang Cao and Wanzhong Zhao \\ College of Energy \& Power Engineering, Nanjing University of Aeronautics and Astronautics, \\ Nanjing 210016, China \\ * Correspondence: zhoujianhao@nuaa.edu.cn; Tel.: +86-84-892-200
}

Received: 9 June 2019; Accepted: 26 June 2019; Published: 27 June 2019

check for updates

\begin{abstract}
Driver perception, decision, and control behaviors are easily affected by traffic conditions and driving style, showing the tendency of randomness and personalization. Brake intention and intensity are integrated and control-oriented parameters that are crucial to the development of an intelligent braking system. In this paper, a composite machine learning approach was proposed to predict driver brake intention and intensity with a proper prediction horizon. Various driving data were collected from Controller Area Network (CAN) bus under a real driving condition, which mainly contained urban and rural road types. ReliefF and RReliefF (they don't have abbreviations) algorithms were employed as feature subset selection methods and applied in a prepossessing step before the training. The rank importance of selected predictors exhibited different trends or even negative trends when predicting brake intention and intensity. A soft clustering algorithm, Fuzzy C-means, was adopted to label the brake intention into categories, namely slight, medium, intensive, and emergency braking. Data sets with misplaced labels were used for training of an ensemble machine learning method, random forest. It was validated that brake intention could be accurately predicted $0.5 \mathrm{~s}$ ahead. An open-loop nonlinear autoregressive with external input (NARX) network was capable of learning the long-term dependencies in comparison to the static neural network and was suggested for online recognition and prediction of brake intensity $1 \mathrm{~s}$ in advance. As system redundancy and fault tolerance, a close-loop NARX network could be adopted for brake intensity prediction in the case of possible sensor failure and loss of CAN message.
\end{abstract}

Keywords: Brake intention; brake intensity; machine learning; electric vehicle; regenerative brake

\section{Introduction}

The intelligent driver assistance system (i-DAS) plays an important role in improving vehicle efficiency, avoiding accidents, and alleviating road congestion. It is a typical human-machine cooperative enhanced intelligent system that is a strong aid to the driver [1]. The i-DAS can be mixed and enhanced through bidirectional information exchange of human-machine intelligence. State monitoring, intention recognition, and driving behavior modeling are essential to human-machine cooperative control [2].

Regenerative braking has become a research focal point of electrified vehicles, since it can recover energy during the routine braking process, thus effectively overcoming range anxiety [3]. However, the variations and state fluctuations among drivers and driving scenarios bring great challenges to the development of the intelligent braking system, which is safety critical. Additionally, the concerns regarding battery health and motor efficiency are integrated into the establishment of a braking control strategy, which makes the algorithm more complicated and computationally intensive. Brake intention 
and intensity are integrated and control-oriented parameters that are crucial to the development of an intelligent braking strategy, such as for an electronic-hydraulic braking (EHB) system [4]. Additionally, efficient recognition of braking intention and intensity are beneficial to shorten the action time of actuators and optimize the regenerative braking strategy [5] and downshift the schedule of the transmission [6] and electric brake force distribution (EBD) [7], hence improving the safety and stability during the braking process. Moreover, the detection of emergency braking is of great importance to driver safety and advanced control techniques, including antilock braking system (ABS), autonomous emergency braking (AEB), and electronic stability program (ESP), which can be triggered in a timely manner $[8,9]$.

Driver perception, decision-making, and control behaviors are easily affected by driving scenarios, psychological, and physiological states, showing the tendency of randomness, personalization, and non-professionalism [10]. With the combination of vehicle operational command and vehicle state parameters, the driver's feet and head positions, and physical and psychological states are obtained through sensors to monitor and analyze the brake intention under various driving scenarios [11-13]. Signal processing, information fusion, and statistical analysis are adopted to enhance the effectiveness of driving behavior analysis. Ji et al. [11] presented a regenerative braking strategy of EV based on the driver's intention identified by a fuzzy recognition method using brake pedal position and speed, and acceleration pedal position and speed. In previous studies [12,13], the driver's electroencephalogram (EEG) and electromyography (EMG) signals were collected and used to classify and recognize the driver's braking intention based on regularization linear discriminant analysis (RLDA).

Supervised and unsupervised machine learning methods, including support vector machine (SVM), dynamic Bayes, neural networks, Markov model, K-means, and Gaussian mixture model (GMM) were employed to recognize brake intention and intensity [14-20]. For instance, cause and effect analysis of accelerator and brake pedal dynamics during an emergency braking scenario was conducted and SVM was employed to recognize emergency braking intention, so as to shorten the driver's response time and reduce the braking distance [14]. Zhao et al. [17] proposed a layering hidden Markov model and adaptive neuro-fuzzy inference system (LHMM/ANFIS) coupled braking intention recognition model, which was applied to develop the Automated Mechanical Transmission (AMT) shift control strategy in order to improve the safety and braking energy recovery efficiency. With consideration of the difficulty of determining the true occurrence of the driver's brake intention, Xing et al. [18] evaluated the performance on brake intention recognition using two different unsupervised machine learning methods, K-means and GMM. The results indicated that GMM yields better performance results, which may recognize the driver's braking intention with a higher accuracy. Zhao et al. [20] further proposed a brake intention recognition algorithm that is coupled with a Gaussian hidden Markov model (GHMM) and radial basis function neural network (RBF).

Brake intensity can be directly indicated by master or wheel cylinder pressure, both of which are control-oriented parameters that are embedded in safety control devices. The current study mainly focuses on the estimation algorithm of master and wheel cylinder pressure. Ding et al. [21] employed least mean square method to predict wheel pressure and tire-road friction based on hydraulic response models developed using control parameters in ABS. Ho et al. [22] proposed a fault tolerant control method in response to pressure sensor failure of an EHB system. A virtual pressure sensor based on a least squares estimation algorithm using motor position and pressure signals during healthy conditions was developed. In a previous study [23], a feed-forward neural network, which was trained with the Levenberg-Marquardt backpropagation algorithm, was developed to estimate master cylinder pressure with high accuracy.

As stated above, EEG and EMG are the most reliable methods to predict brake intention, but brain signal extraction is a particularly complex process and extremely expensive experimental equipment is required, which is apparently not ready for practical use [24,25]. The brake intention and intensity are usually bound and recognized according to the pedal displacement and the state parameters of the vehicle (velocity, acceleration, etc.) using machine learning methods. Actually, braking action 
indicated the driver's response to the surrounding traffic conditions, which is closely related to the external environment, such as the distance between the preceding vehicle, the road curvature and slope, the traffic light, and the pedestrian. However, the above factors are not directly associated with the braking action and present discontinuity during the prediction of brake intention and intensity. Therefore, if external factors are considered comprehensively, the classification and prediction of brake intention and intensity will be disturbed. Nevertheless, most of the existing studies have paid particular attention to the accuracy of the proposed model for brake intention and intensity recognition, which was generally capable of online estimation or one-step prediction at most. However, the investigations on multi-step or long term prediction of brake intention and intensity are essential to the development of an intelligent braking system to enhance brake safety and efficiency, and the related studies are scarce.

This paper is organized as follows. The experimental data collection method is introduced in Section 2. Then, the brake intention and intensity prediction framework is presented in Section 3. In Section 4, unsupervised and supervised machine learning methods, namely fuzzy C-means (FCM) and random forest (RF), respectively, are coupled and employed to train the brake intention prediction model. In Section 5, a dynamic recurrent neural network, nonlinear auto-regression with exogenous input (NARX) featured with feedback and embedded memory is adopted to predict brake intensity. Lastly, conclusions are summarized in Section 6.

\section{Experimental Data Collection}

\subsection{Test Vehicle and Its Braking System}

The test vehicle used in this study is a forward wheel drive (FWD) Electric Vehicle (EV) (model T15EV) made by Chery automobile co. LTD, China. Figure 1 presents the block diagram of its electro-hydraulic composite braking system. The electronic vacuum booster, pressure accumulator, and pedal simulator are not related to this study, hence they are not depicted. $T_{m f l}$ and $T_{m f r}$ refer to the regenerative braking torque of the front left and right wheels, respectively. Correspondingly, $T_{h f l}, T_{h f r}$, $T_{h r l}$, and $T_{h r r}$ represent the hydraulic braking (frictional) torque of four wheels. Signals from sensors, actuators, and state observers contain brake pedal displacement, vehicle velocity, road adhesion coefficient, master cylinder pressure, etc., and the messages transferred via CAN bus, including State of Charge (SOC) from the battery management system (BMS), motor torque, and speed from the motor control unit (MCU), are sent to the vehicle control unit (VCU). According to the information collected, the VCU calculates the regenerative and hydraulic (frictional) braking torque and sends the required torque signals to the MCU and the hydraulic control unit (HCU), respectively.

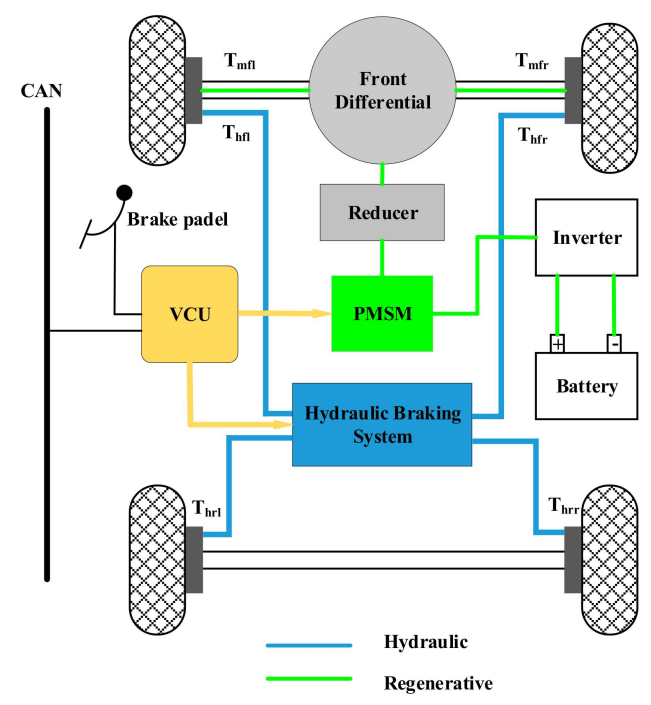

Figure 1. Block diagram of electro-hydraulic composite braking system of the test electric vehicle. 


\subsection{Data Collection}

It is noted that the brake pedal displacement and its gradient, the vehicle velocity and acceleration, and wheel cylinder pressure were widely used in previous studies $[20,23]$ for brake intention and intensity recognition. In order to reflect the parameters that potentially affect the prediction performance of brake intention and intensity, master cylinder pressure (MCP), battery current and voltage, motor speed and torque, etc., are selected for model training, which will dynamically vary during the braking process of an electrified vehicle. The signals as predictors used for model training are listed in Table 1. STD represents the standard deviation of the predictor during the preceding $3 \mathrm{~s}$.

Table 1. Predictors' used for model training.

\begin{tabular}{cccc}
\hline No. & Predictors & No. & Predictors \\
\hline 1 & Velocity $(\mathrm{km} / \mathrm{h})$ & 8 & Battery current gradient $(\mathrm{A} / \mathrm{s})$ \\
2 & Mean velocity $(\mathrm{km} / \mathrm{h})$ & 9 & Battery voltage $(\mathrm{V})$ \\
3 & Standard deviation of velocity $(\mathrm{km} / \mathrm{h})$ & 10 & Battery volatge gradient $(\mathrm{A} / \mathrm{s})$ \\
4 & Acceleration $\left(\mathrm{m} / \mathrm{s}^{2}\right)$ & 11 & State of Charge $(\mathrm{SOC})(\%)$ \\
5 & Mean acceleration $\left(\mathrm{m} / \mathrm{s}^{2}\right)$ & 12 & Motor torque $(\mathrm{N} . \mathrm{m})$ \\
6 & Standard deviation of acceleration $\left(\mathrm{m} / \mathrm{s}^{2}\right)$ & 13 & Motor speed (rpm) \\
7 & Battery current $(\mathrm{A})$ & 14 & Master cylinder pressure $(\mathrm{MPa})$ \\
\hline
\end{tabular}

As shown in Figure 2a, the cyclic test route started and ended at Nanjing University of Aeronautics and Astronautics (marked by A) and passed by four different universities located in Nanjing (marked by B, C, D, and E, respectively). The test route mainly contains urban and rural road types. Highways were intentionally avoided as much as possible since the driving condition was smoother in urban and rural conditions, where braking maneuvers seldom occurred. The overall distance and the average velocity of the test route are around $29 \mathrm{~km} / \mathrm{h}$ and $60 \mathrm{~km}$, respectively. The required signals listed in Table 1 were collected at a sample frequency of $10 \mathrm{~Hz}$ from a control area networks (CAN) bus using CANcaseXL (Vector co., Ltd., Germany). A total of 75411 data sets were collected. In Figure 2b, it can be seen that the velocity and MCP profiles present no periodic acceleration or deceleration compared with standard driving conditions, which reflects the fluctuation of vehicle speed and braking intensity with different degrees of road congestion under real driving conditions.

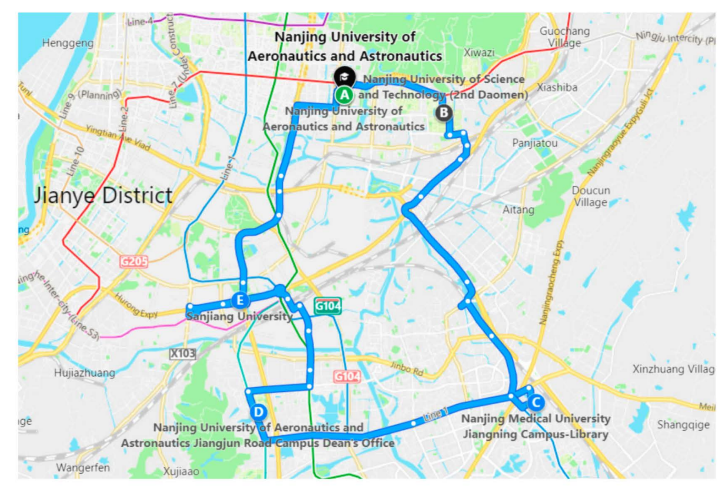

(a)

Figure 2. Cont. 


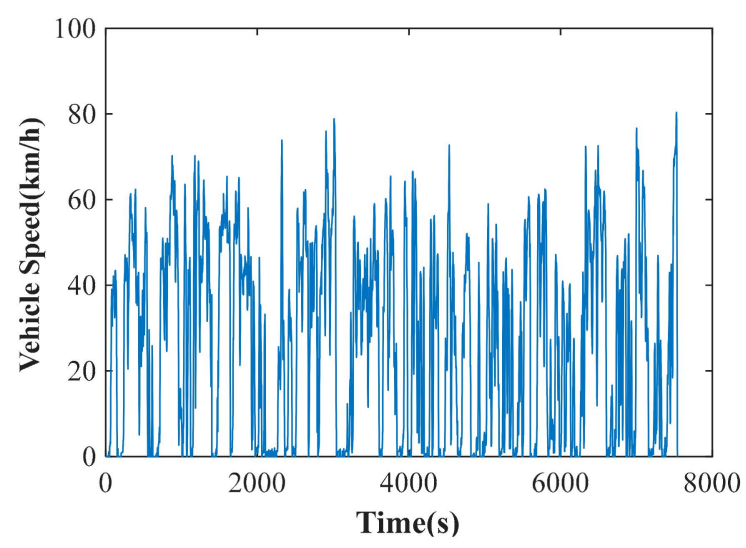

(b)

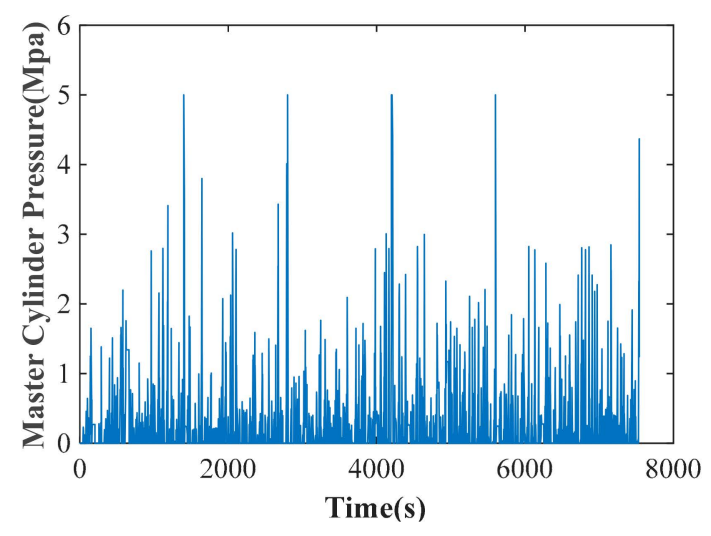

(c)

Figure 2. The test routes and signal profiles. (a) The cyclic test route $(A \rightarrow B \rightarrow C \rightarrow D \rightarrow E \rightarrow A)$. (b) Vehicle velocity profile. (c) Master cylinder pressure profile.

\section{Hybrid Machine Learning Framework}

Figure 3 presents the systematic framework of brake intention and intensity prediction using a hybrid machine learning approach. The main idea is to build a brake intention and intensity prediction model through a composite machine learning approach and then explore and verify its feasibility of producing a prediction horizon before the instant when a driver intends to brake under various drive scenarios with different brake intensities.

Various parameters from sensors, actuators, state observers, and the CAN bus can be obtained to analyze the driver's braking maneuvers. Brake intention is usually classified by a series of thresholds of MCPs for a rule-based strategy of regenerative braking, which is robust and reliable. However, pre-calibration of MCP thresholds are necessary for such a strategy, which are not able to deal with different driving styles and driving scenarios, hence it is suboptimal and less helpful for developing a personalized braking system [19]. Besides, MCP is an important control-oriented parameter that is useful and efficient in EBD, ABS, ESP, etc., [8,9]. Therefore, MCP is initially fed to FCM for offline training and brake intention labeling. Then, the MCP data sets four of its own labels, representing slight, medium, intensive, and emergency braking. After this, 14 kinds of predictors as data sets, including $\mathrm{MCP}$, velocity, acceleration, batter current, etc., and 4 labels from FCM as target values are used for offline training of the RF model, which follows the concept of sensor fusion. Moreover, the ReliefF algorithm for rank importance analysis of predictors is integrated and the predictors that have less weight are removed in order to simplify the training model and save the computational time. During the training, the target values (as labels) and input data sets are intentionally preprocessed to 
have a time gap, so as to form a prediction horizon and achieve the prediction effect on brake intention. A similar method was also adopted in a previous study [26]. Finally, the trained RF model can be employed for online prediction of brake intention within a prediction horizon.

On the other hand, brake intensity is usually recognized as a discrete state which is treated as a threshold value using MCP, as mentioned [previously. With consideration of the natural continuity of brake intensity during normal braking maneuvers, especially under urban and rural road conditions, NARX, as a dynamic recurrent neural network with feedback and memory ability, is adopted to predict brake intensity. Similarly, 14 kinds of predictors were collected as data sets for offline training of the NARX model and the RReliefF algorithm is adopted to remove the predictors with low weight. Since NARX is efficient in prediction of sequential or time-varying patterns, particular attention was paid to the effective prediction horizon of the brake intensity that can be predicted with acceptable accuracy. Besides, system redundancy and fault tolerance are important features bound by intelligent braking control. Since MCP is a critical signal related to braking safety, the proposed NARX model can also play the role of a virtual sensor for online prediction of MCP in the case of sensor failure. Therefore, even if the ground-truth signal of MCP is missing, the braking intention and intensity prediction model can still work properly.

The algorithms are implemented in a computer with the MATLAB 2017b platform. The processor of the computer is an Intel Core i5 CPU, which supports four cores and eight threads for parallel computing, while the RAM equipped is a $16 \mathrm{G}$ unit.

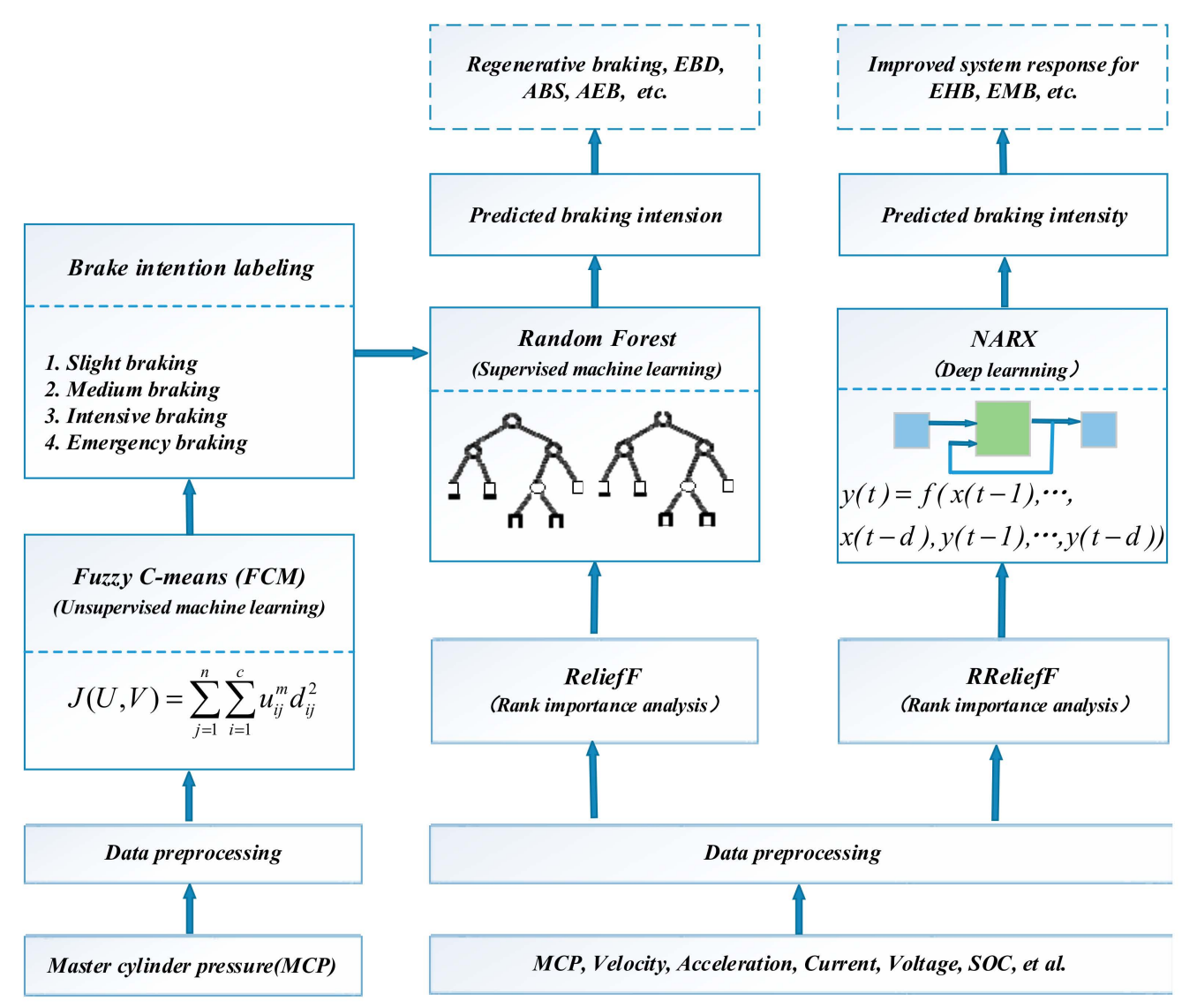

Figure 3. Systematic framework of brake intention and intensity prediction using a hybrid machine learning approach. 


\section{Brake Intension Prediction}

\subsection{FCM-Based Brake Intention Labeling}

\subsubsection{Fuzzy C-means Clustering Algorithm}

The FCM algorithm is adopted in this study to label the brake intention. FCM is an unsupervised machine learning algorithm that does not need training labels before clustering, only the number of clusters. The FCM algorithm is different from K-means, GMM, and other hard clustering algorithms. It is a soft clustering algorithm that allows each sample to belong to multiple clusters with varying degrees of membership, and hence more objectively reflects the clustering results.

FCM is based on the minimization of the following objective function [27]:

$$
J_{m}=\sum_{j=1}^{n} \sum_{i=1}^{c} u_{i j}^{m} d_{i j}^{2}
$$

where $n$ is the number of data sets; $c$ is the number of clusters; $m$ is fuzzy partition matrix exponent for controlling the degree of fuzzy overlap, which refers to how fuzzy the boundaries between clusters are; $d_{i j}$ is the distance between data set $x_{i}$ to the center of cluster $c_{j} ; u_{i j}$ is the degree of membership of $x_{i}$ in the $c_{j}$.

FCM performs the following steps during clustering [27]:

(1) Randomly initialize the cluster membership values, $u_{i j}$.

(2) Calculate the cluster centers:

$$
c_{j}=\frac{\sum_{j=1}^{n} u_{i j}^{m} x_{i}}{\sum_{j=1}^{n} u_{i j}^{m}}
$$

(3) Update $u_{i j}$ according to the following:

$$
u_{i j}=\frac{1}{\sum_{k=1}^{c}\left(\frac{\left\|x_{i}-c_{j}\right\|}{\left\|x_{i}-c_{k}\right\|}\right)^{\frac{2}{m-1}}}
$$

(4) Calculate the objective function, $J_{m}$.

(5) Repeat steps 2-4 until $J_{m}$ improves by less than a specified minimum threshold or until after a specified maximum number of iterations.

MCP is chosen as the sole input of FCM and the clustering number is set to 4, on behalf of slight, medium, intensive and emergency brake intensions.

\subsubsection{Brake Intention Labeling Results}

As shown in Figure 4, the range of MCP is $0-0.22 \mathrm{MPa}$, which refers to slight braking and accounts for $73.88 \%$ of the total data sets, indicating that no braking control should be adopted for the vehicle. The vehicle may drive on a bumpy road and that the slight braking is caused by the loosening of the brake pedal. The range of $\mathrm{MCP}$ is $0.22-0.87 \mathrm{MPa}$, which represents moderate braking and accounts for $17.98 \%$ of the total data sets. The distance between the vehicle and the forward vehicle is considered to be long enough. Riding comfort and energy recovery efficiency can be given priority during braking control. In comparison, when the range of MCP moves to $0.87-2.92 \mathrm{MPa}$, which accounts for $7.56 \%$ of the total data sets, it is considered that the distance between the forward vehicle is almost within an absolute safety distance. The driver's desired declaration is dominant during braking control. Lastly, 
emergency braking is labeled when MCP exceeds 2.92MPa. However, such scenarios only account for $0.58 \%$ of the data sets. In these moments, ABS, AEB, and other emergency control systems should be involved for safety control.

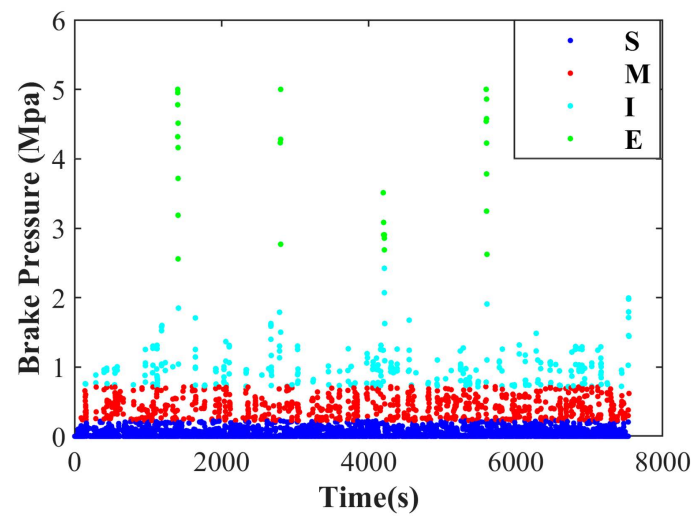

Figure 4. Labeling results of different brake intentions.

Figure 5 randomly captures a window of probabilities of 20 data sets. The green, blue, black, and red circles represent the probabilities of slight, medium, intensive, and emergency brake, respectively. The sum of the four probabilities in each group equals to 1 . It can be found that the probabilities of groups 200-204, 205-208, and 219-220, belonging to slight braking, are 1, hence the other three circles on the X-axis overlap. Among them, special attention should be paid to data set 216, which belongs to intensive and emergency braking with probabilities of $41.58 \%$ and $45.55 \%$, respectively. The probabilities of the two brake intentions are close and the emergency braking label is set with consideration of driving safety. Actually, those close probabilities of two clusters are rare and probably occur at the threshold of each cluster, but do not pose a threat to the safety and stability of the vehicle.

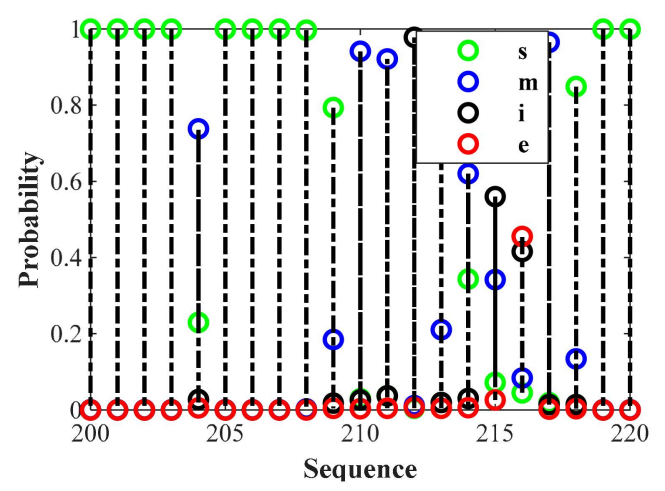

Figure 5. Probability result fragments of each brake intention label.

\subsection{ReliefF Rank Importance Analysis}

One of the primary intentions in this study is to explore the available signals (predictors) via CAN bus that may potentially affect the brake intention prediction result. However, those predictors as listed in Table 1 may involve feature interaction and are less appropriate as qualified predictors. The ReliefF algorithm has been widely recognized as a feature subset selection method that is applied in a prepossessing step before the model is learned [28]. Therefore, the rank importance of each predictor is analyzed using the ReliefF algorithm before the training of the brake intention prediction model.

ReliefF randomly selects a sample $S$ from sample sets $Q$ and then searches for $j$ of its nearest neighbors from the same class, namely hitting the $\mathrm{NH}_{j}$, and also $j$ of its nearest neighbors from each of 
the different classes, missing $N M_{j}$. Feature weight $W[X]$ for all features $X$ is updated depending on the values of $S, N H_{j}$, and $N M_{j}$ using the formula listed as follows [29]:

$$
W[x]=W[x]-\sum_{j=1}^{k} \operatorname{diff}\left(X, S, N H_{j}\right) /(m k)+\sum_{C \neq C \text { Class }(S)}\left[\frac{P[C]}{1-P[\operatorname{Class}[S]]} \sum_{j=1}^{k} \operatorname{diff}\left[X, S, N M[C]_{j}\right]\right] /(m k)
$$

where $m$ refers to the interaction number, $P(C)$ stands for the probability of $C$ class, $\operatorname{Class}(S)$ is the class of sample $S, \operatorname{diff}\left(X, S, N M(C)_{j}\right)$ represents the distance between sample $S$ and $N M(C)_{j}$ related to feature $X$.

The rank importance of each predictor is shown in Figure 6. It can be found that MCP, mean and transient velocity, and mean and transient acceleration have higher rank importance weight than others. Since the motor speed and torque directly influence vehicle velocity and acceleration, their weights are also high. Meanwhile, the battery current and voltage tend to be changed during regenerative braking, hence they present relative high rank importance weight. However, the gradient of the battery current and voltage show less weight. To conclude, battery current gradient, voltage gradient, and STD of acceleration are removed during RF training.

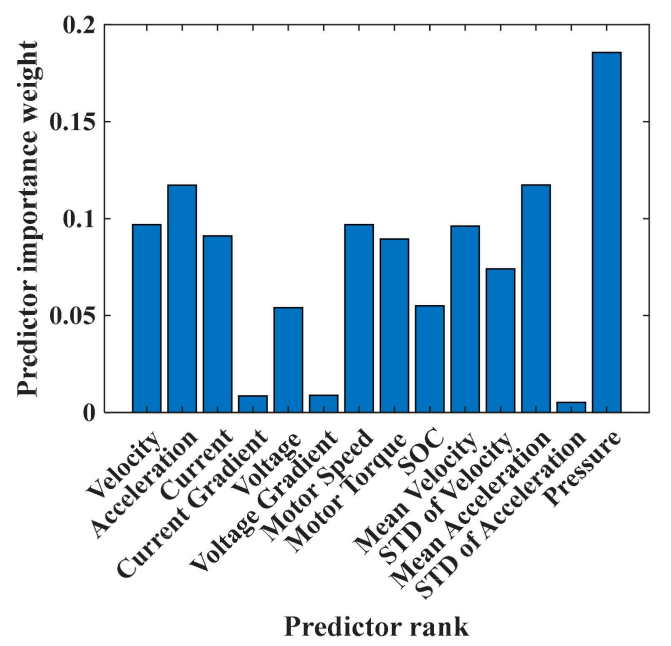

Figure 6. Rank importance of each predictor during brake intention prediction using ReliefF.

\subsection{RF Based Brake Intention Prediction}

\subsubsection{Random Forest Classification Algorithm}

$R F$ is employed in this study to predict the brake intention. RF is a supervised machine learning algorithm that needs predefined labels as target inputs. The main steps of the RF algorithm are as follows [30]:

(1) The bootstrap sampling method is used to get $n$ of training samples from the original training samples.

(2) Each training sample is trained to generate a single decision tree, and the features of the decision tree are randomly selected. The splitting rule of the decision trees is according to the CART (Classification and Regression Tree) algorithm and the minimum principle of the Gini coefficient. The Gini coefficient can be expressed as follow:

$$
\operatorname{Gini}(P)=\sum_{k=1}^{k} P_{k}\left(1-P_{k}\right)=1-\sum_{k=1}^{k} p_{k}^{2}
$$

where $P_{k}$ is the probability of occurrence of category $k$. 
(3) Each decision tree is trained to generate a prediction result according to the randomly selected characteristics of the sample, which reduces the effects of overfitting and improves generalization.

(4) All of the predicted results of the decision tree are gathered to determine the final predicted results by voting.

Additionally, Support Vector Machine (SVM), K nearest neighbors (KNN), decision tree, and AdaBoost Trees are widely used in classification problems and their performance on brake intention prediction are compared with RF. Table 2 shows the comparison of different classification algorithms applied in this study. In general, the accuracy of the RF algorithm yields the best performance with an accuracy of $97.3 \%$, which is obviously higher than the other four algorithms. The accuracies of the decision tree and AdaBoost Trees are $94.8 \%$ and $94.1 \%$, respectively. KNN obtains the lowest accuracy and SVM needs the longest training time.

The KNN algorithm mainly relies on the limited neighboring samples rather than the method of identifying the class domain to determine the training sets, and the output is not interpretable. Due to the unequal number and imbalance of samples in each training set for brake intention prediction, the accuracy of KNN performs the worst. When dealing with multi-classification problems, SVM needs to construct multiple combinations of binary-class SVMs, which is time consuming and inefficient. AdaBoost Trees and RF are typical ensemble learning algorithms that can improve the generalization ability of the model in comparison to a simple decision tree. Meanwhile, an ensemble learning algorithm can avoid multiple training classifiers in classification in comparison to SVM, which saves memory and training time, and simplifies the training model. The main difference between AdaBoost Trees and $\mathrm{RF}$ is the sampling method. The former adopts the boosting sampling method, which is in serial, and every sampling process depends on the last one, hence is time consuming. The boosting sampling method is also weighted and the training sets with various weights may affect the classification results. RF employs the bagging sampling method, which is randomized, independent, and in parallel. The correlation between each decision tree tends to be low and the overfitting problem can be avoided. Therefore, the RF algorithm is employed to predict brake intention.

Table 2. Comparison of different classification algorithms.

\begin{tabular}{ccc}
\hline Classification Algorithm & Accuracy (\%) & Training Time(s) \\
\hline Support Vector Machine(SVM) & 93.10 & 23.08 \\
K nearest neighbors (KNN) & 91.40 & 7.13 \\
Decision Tree & 94.80 & 9.65 \\
AdaBoost Trees & 94.10 & 17.84 \\
Random Forest & 97.30 & 11.26 \\
\hline
\end{tabular}

\subsubsection{Performance Analysis of Brake Intention Prediction}

The performance analysis of brake intention recognition can be divided into two categories. One is to investigate how many times successful and unsuccessful brake intention recognitions can be obtained among all the data sets, which can be expressed as a confusion matrix. The other is to verify how long the prediction horizon can predict the brake intention with acceptable accuracy. It is known that a longer predictive horizon for brake intention is helpful to establish a corresponding control strategy under different driving scenarios, but this doubtlessly sacrifices the prediction accuracy. Therefore, there is a time gap that is treated as the prediction horizon between the label given by FCM and the predictors. In other words, the target values that feed the RF model for training exist in a future moment, so as to achieve brake intention prediction, rather than brake intention recognition [26]. In order to ensure accuracy, different prediction horizons of the RF model for braking intention prediction are analyzed using the confusion matrix, as shown in Figure 7, where 1, 2, 3, and 4 represent slight, medium, intensive, and emergency braking, respectively. Since there are large numbers of data sets, the overall prediction accuracy tends to be extremely high and the incorrect prediction cannot be 
clearly distinguished. In total 1900 samples, which are generally equal to the data sets available during standard driving cycles [23], are randomly selected for verification, including 1493 slight samples, 300 medium samples, 89 intensity samples, and 18 emergency samples.

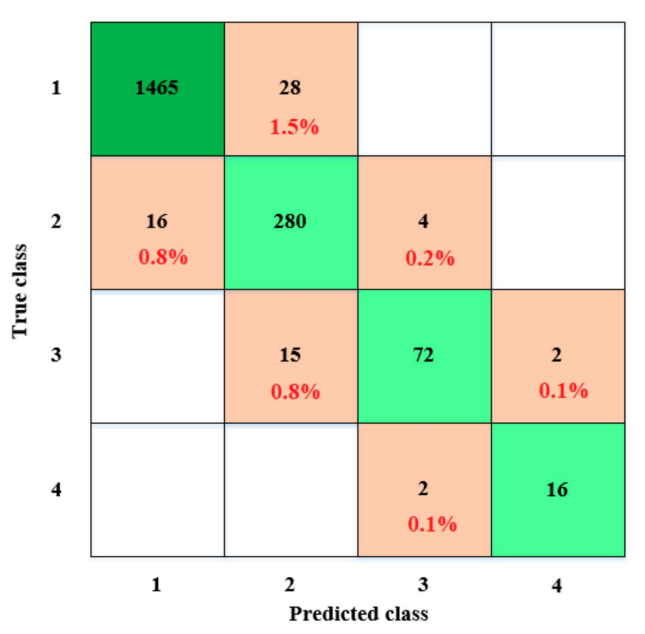

(a)

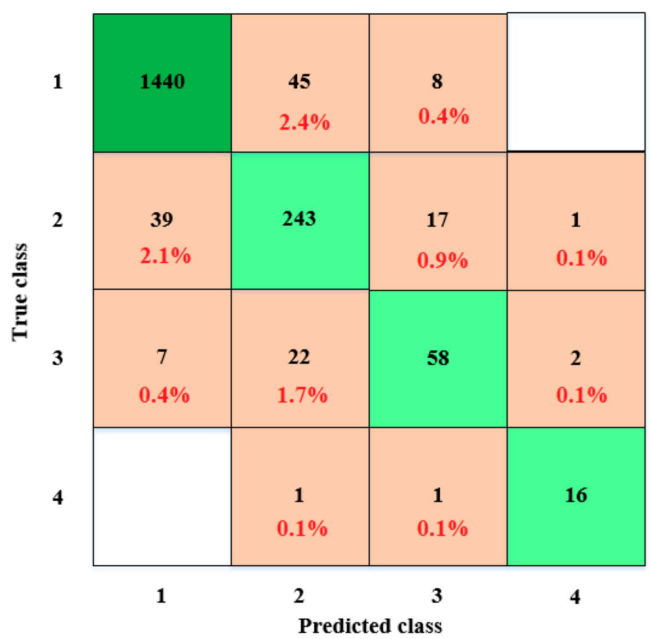

(b)

Figure 7. Confusion matrix of brake intention using random forest. (a) Prediction horizon (time) $=0.5 \mathrm{~s}$; (b) prediction horizon (time) $=1 \mathrm{~s}$.

Figure 7a shows the RF predicted results presented by the confusion matrix, with a prediction horizon of $0.5 \mathrm{~s}$. The overall prediction accuracy is $96.5 \%$. Specifically, there are 1493 and 1465 data sets with actual and predicted values of slight braking, respectively, which indicates an accuracy of $98.1 \%$. Similarly, the prediction of medium, intensive, and emergency braking intention can achieve accuracies of $93.3 \%, 80.9 \%$, and $88.9 \%$, respectively. Among the 57 groups of incorrect predictions, the brake intention levels are close. For instance, 2 emergency braking classes are incorrectly predicted to be intensive braking, among 18 in total. Therefore, this has a small impact on braking safety.

Figure $7 \mathrm{~b}$ shows the confusion matrix of the prediction results when the prediction horizon is $1 \mathrm{~s}$. Among 1900 samples, 1767 samples have correct prediction results, with an overall accuracy of $92.5 \%$. However, among the error predictions, there are two or three possible outcomes between the predicted and the actual brake intentions, which will have a negative impact on the safety of the vehicle. For instance, there will be a safety hazard if emergency braking is incorrectly predicted to be medium braking or if intensive braking is incorrectly predicted to be slight braking. Therefore, when the brake intention is predicted based on the FCM-RF coupled hybrid machine learning algorithm, the prediction horizon can be selected as $0.5 \mathrm{~s}$.

\section{Brake Intensity Prediction}

\subsection{RReliefF-Based Rank Importance Analysis}

The predictors as shown in Table 1 are also applied for brake intensity prediction. Unlike brake intention, the brake intensity is usually considered to be continuous in real driving conditions, hence hits and misses that applied in Relief cannot be used. As an update, RReliefF introduces a kind of probability that can be modelled with the relative distance between the predicted values of two samples, which can be computed as follows [29]:

$$
\begin{gathered}
\omega_{d C}=\omega_{d C}+\operatorname{diff}\left[\varepsilon[\cdot], S, N H_{j}\right] \cdot d(i, j) \\
\omega_{d X}[X]=\omega_{d X}[X]+\operatorname{diff}\left[X, S, N H_{j}\right] \cdot d(i, j)
\end{gathered}
$$




$$
\omega_{d C \& d X}[X]=\omega_{d C \& d X}[X]+\operatorname{diff}\left[\varepsilon[\cdot], S, N H_{j}\right] \cdot \operatorname{diff}\left[X, S, N H_{j}\right] \cdot d(i, j)
$$

where $\omega_{d c}, \omega_{d X}[X]$, and $\omega_{d C} \& d X[X]$ are the weights for different prediction values $\tau(\cdot)$, different features, and different predictions and features, respectively; $d(i, j)$ takes into account the distance between the two samples $S$ and $\mathrm{NH}_{j}$. Feature weight $W[X]$ can be finally computed as follow [29]:

$$
W[X]=\frac{\omega_{d C \& d X}[X]}{\omega_{d C}}-\frac{\omega_{d X}[X]-\omega_{d C \& d X}[X]}{m-\omega_{d C}}
$$

Figure 8 presents the rank importance analysis results of 14 different predictors, which exhibit certain differences in comparison to the rank importance of predictors used for brake intention prediction, as shown in Figure 6. Specifically, MCP obtains the highest weight, which is reasonable since it directly reflects the intensity of the driver who pressed on brake pedal. It is also noted that the weight of the battery current, the current gradient, and voltage gradient are negative, which may have a negative impact on the prediction performance of brake intensity. The predictors that indicate the transient kinetics of the vehicle have relatively higher weights, such as the STD of velocity, acceleration, and STD of acceleration. The influence of battery current gradient, motor speed and torque, and SOC are positive and consistent with the trend of brake intensity when regenerative braking is triggered. However, their contribution of brake intensity prediction is inconspicuous. In order to save computational time and simplify the model, those predictors with very small weights are omitted.

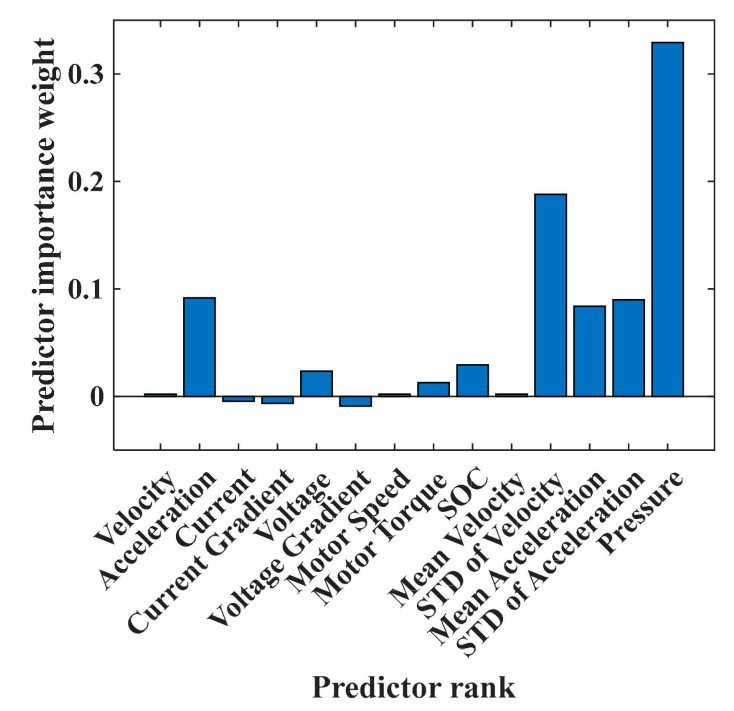

Figure 8. Rank importance analysis of each predictor during brake intensity prediction using RReliefF.

\subsection{NARX Network-Based Brake Intensity Prediction}

\subsubsection{NARX Network}

Static neural networks (SNN) have been successfully applied to deal with time series prediction and nonlinear modeling tasks, including brake intensity estimation. However, multi-step prediction of brake intensity is difficult to realize using SNN, which is attributed to the well-known long-term dependencies [31], which means that the desired output of a system at time $\mathrm{T}$ depends on inputs presented at times $t(t<T)$. Especially, when using a gradient-decent training algorithm, such as backpropagation, a problem called the vanishing gradient is essential to the occurrence of long-term dependencies [32]. Since the braking maneuvers are strongly related to driver's braking style and driving scenario, brake intensity is supposed to be influenced by the historical actions taken by the driver, hence exhibiting long-term dependencies. Therefore, NARX, as a dynamic recurrent neural network (DRNN) with feedback and memory functions, is applied in this study for brake intensity 
prediction. Particular attention is paid to its learning ability regarding long-term dependencies and its sensitivity to the vanishing gradient, even when using the gradient-decent training algorithm.

The defining equation for the NARX model is presented as follows [33]:

$$
y(t)=f\left(\mathrm{u}(t-1), \ldots, u\left(t-n_{u}\right), y(t-1), \ldots, y\left(t-n_{\mathrm{y}}\right)\right)
$$

where $u(t)$ and $y(t)$ refer to the input and output of the network at time $t ; n_{u}$ and $n_{y}$ represent the input and output memory orders, respectively. The function $f$ is a nonlinear function that can be approximated by a multilayer perceptron (MLP) and the resulting connectionist architecture is then formed by a NARX recurrent neural network.

Figure 9 illustrates the structure diagram of the proposed NARX network, where $d=n_{u}=n_{y}$. The neural network is composed of three layers: the input layer, hidden layer, and output layer [34]. The number of hidden layers of the neural network is 1 in this paper. The activation function $f_{1}$ is a tansig function, which calculates the output $H_{k}$ of the hidden layer neuron using the following formula [34]:

$$
\left.H_{j}=f_{1}\left(\sum_{i=1}^{n} w_{i j} u_{j}+b_{i}\right)\right), j=1,2, \ldots \ldots, l
$$

where $w_{i j}$ denotes the connection weight between the input node $i$ and the $j$ th node of hidden layer; $l$ is the number of neurons in the hidden layer; $b_{j}$ is the connection weight for the bias term.

The activation function $f_{2}$ is a purline function that calculates the output $y_{k}$ using the following formula [34]:

$$
\left.y_{k}=f_{2}\left(\sum_{j=1}^{l} H_{j} w_{j k}+b_{j}\right)\right)
$$

where $w_{j k}$ refers to the connection weight between the $j$ th node of the hidden layer and output node $k$.

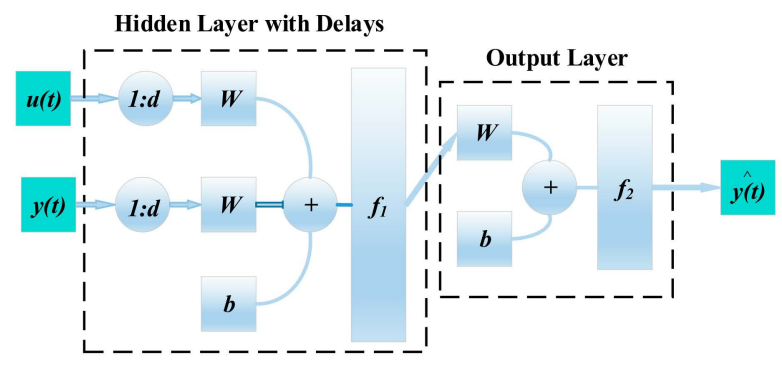

(a)

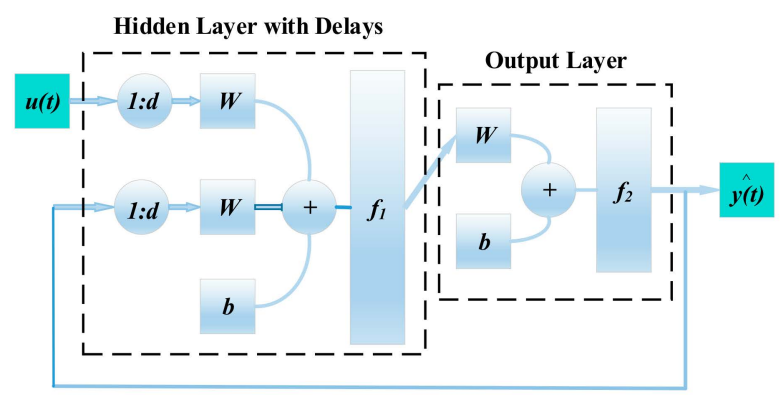

(b)

Figure 9. Typical configurations of the nonlinear autoregressive with external input (NARX) network during training. (a) Open-loop NARX network; (b) close-loop NARX network. 
In order to evaluate the training results of the NARX network, two evaluation indices-the mean square error (MSE) and correlation coefficient (R)-were adopted. MSE indicates the error between the training output value and the expected target value, which is computed by:

$$
M S E=\frac{1}{N} \sum_{i=1}^{N}(X(t)-Y(t))^{2}
$$

$R$ reflects the correlation between the training output value and the expected target value, which can be computed by:

$$
R=\frac{\sum_{i=1}^{N}(X-\bar{X})(Y-\bar{Y})}{\sqrt{\sum_{i=1}^{N}(X-\bar{X})^{2}} \sqrt{\sum_{i=1}^{N}(Y-\bar{Y})^{2}}}
$$

where $N$ is the number of total training sets, $X(t)$ refers to the expected output values, and $Y(t)$ is the actual training output values.

\subsubsection{Performance Analysis of Brake Intensity Prediction}

In order to evaluate the impact of the training algorithm on prediction performance for open-loop NARX, various backpropagation algorithms, including the scaled conjugate gradient (SCG), one-step secant (OSS), quasi-Newton (QN), gradient descent with momentum (GDM), Levenberg-Marquardt (LM), and Bayesian regularization (BR) methods were adopted and compared. The prediction performance of the brake intensity of the selected training algorithm is shown in Table 3. It can be seen that BR yields the lowest MSE and R among different algorithms but sacrifices training time. LM is the most suitable training algorithm with high accuracy and correlation and low training time. The LM algorithm is a combination of gradient descent and Gauss-Newton methods. The LM algorithm takes advantages of an approximation form of the Hessian matrix in the following Newton-like update [35]:

$$
\Delta w=-\left[J[w]^{T} J[w]+\mu I\right]^{-1} J(w)^{T} e(w)
$$

where $e(w)$ is the error vector, which is used to compute error function $E(w)$; J(w) refers to the Jacobian matrix of $e(w)$ and $J(w)^{T}$ is its transpose form; $J(w)(w)^{T}$ stands for approximated Hessian matrix; $I$ is the identity matrix; $\mu$ is the learning constant, which is adjusted based on the error in each iteration to find the minimum $E(w)$.

Table 3. Performance of brake intensity recognition using the close-loop NARX network with different training algorithms.

\begin{tabular}{cccc}
\hline Training Algorithms & Mean Square Error & R & Training Time (s) \\
\hline Scaled Conjugate Gradient (SCG) & 0.003126 & 0.981 & 9 \\
One-step Secant (OSS) & 0.003779 & 0.979 & 25 \\
Quasi-Newton (QN) & 0.001714 & 0.988 & 10 \\
gradient descent with momentum (GDM) & 0.126163 & 0.925 & 5 \\
Levenberg-Marquardt (LM) & 0.001408 & 0.991 & 19 \\
Bayesian Regularization (BR) & 0.001248 & 0.992 & 37 \\
\hline
\end{tabular}

The influences of input and output memory orders and number of neurons on the performance of brake intensity recognition using NARX networks are prohibited in Figure 10. It can be found that MSE and $\mathrm{R}$ decrease and increase with memory order of 2, respectively. A further increase the memeory order will not contribute to the improvement of prediction performance. Although the embedded memory of the NARX network has a strong impact on its dynamical behavior and is essential to improving its learning ability, the computational time will sharply increase with the increase of its 
memory order and its number is set to 5 . In other words, data within $0.5 \mathrm{~s}$ should be encapsulated in the neural network because the sampling frequency is $10 \mathrm{HZ}$. In order to avoid overfitting or underfitting, the influnence of the neuron number is analyzed. It can be seen that overfitting tends to occur when the number of neurons exceeds 100. When the number of neurons increases from 10 to 80, no apparent variation of MSE and R can be found. With consideration of model simplicity, the number of neuron is set as 20 .
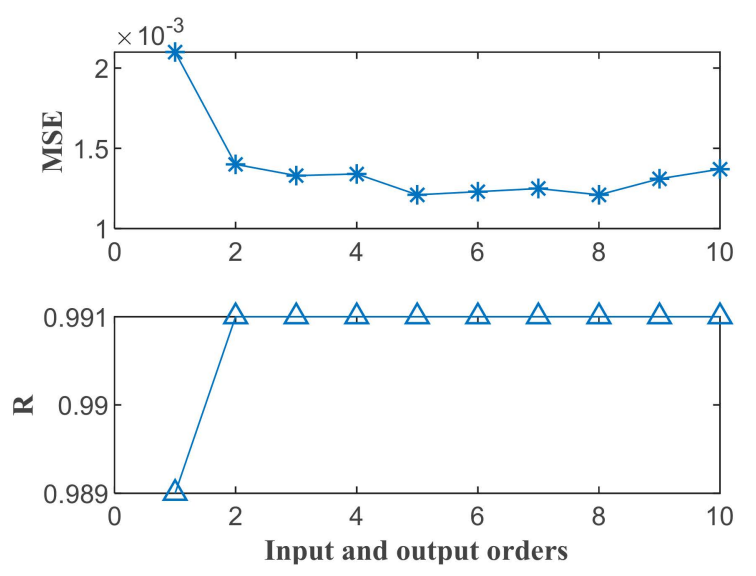

(a)
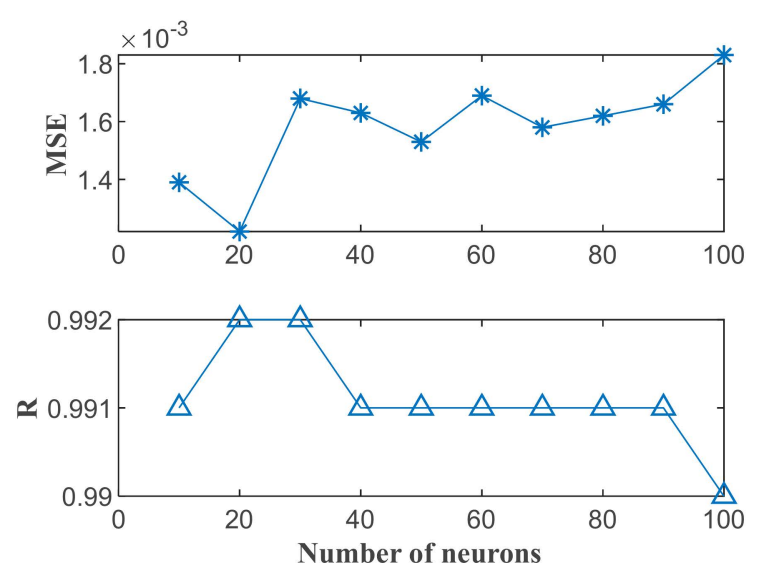

(b)

Figure 10. Performance indices of brake intensity recognition using the close-loop NARX network with different memory orders and numbers of neurons. (a) Performance with different memory orders; (b) Performance with different numbers of neurons.

Figure 11a illustrates the linear regression performance of the trained model and the validation regression result $R$ is 0.989 . The best linear fit (green solid line) is very close to the perfect fit (black dashed line), which indicates that the simulation accuracy of the NARX neural network is very high. Figure $11 \mathrm{~b}$ presents the brake intensity recognition results using the close-loop NARX network with 5 memory orders and 20 neurons within 1000 randomly selected samples; the close-loop NARX network has 5 memory orders, meaning data should be encapsulated in the neural network within $0.5 \mathrm{~s}$. In general, the error between the predicted value and the target value is almost 0 and the error exceeds 0.1 only when the braking intensity changes suddenly. Although a small portion of the predicted brake intensity is negative, those predicted MCPs are all near zero MPa can be recognized as a glide or acceleration state and will not affect the energy management system and brake control system. Therefore, the NARX network can be utilized as a powerful brake intensity recognition model with high accuracy. 


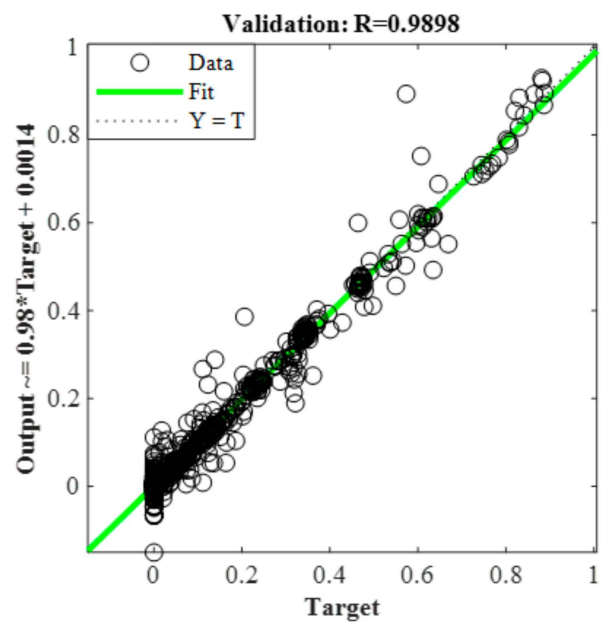

(a)

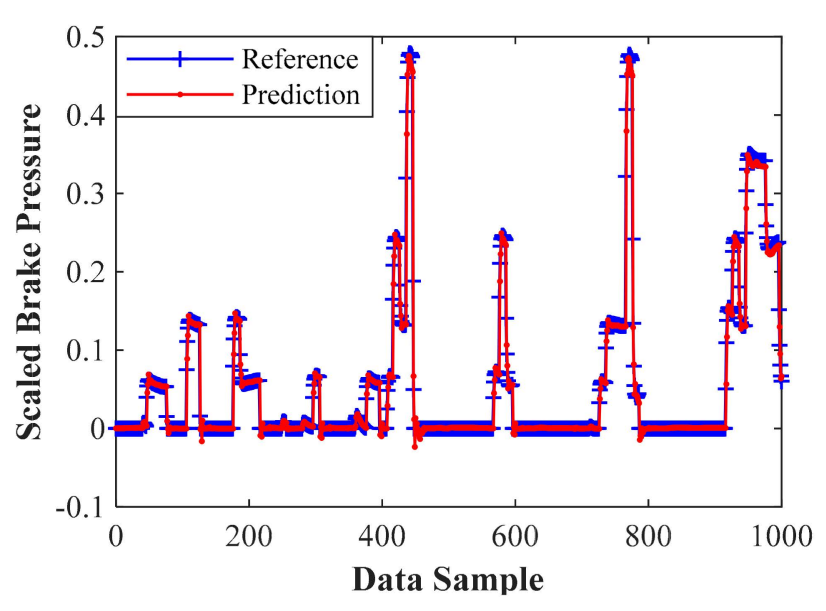

(b)

Figure 11. Brake intensity recognition results using the close-loop NARX network with 5 memory orders and 20 neurons. (a) Linear regression fit; (b) brake inensity recognition results.

As a typical Dynamic Recurrent Neural Networks (DRNN) the configuration of the NARX network will influence its prediction performance. The NARX network can be generally trained in open-loop and close-loop modes, as shown in Figure 9 [36]. The performance comparison of brake intensity prediction using open-loop and close-loop NARX networks is shown in Figure 12. Both open-loop and close-loop NARX networks employ the LM training algorithm. Additionally, the performance of a typical SNN-the LM backpropagation (LMBP) neural network - is given as the baseline. Particular attention was paid to the performance variation, along with the increase of prediction horizon among the three models.

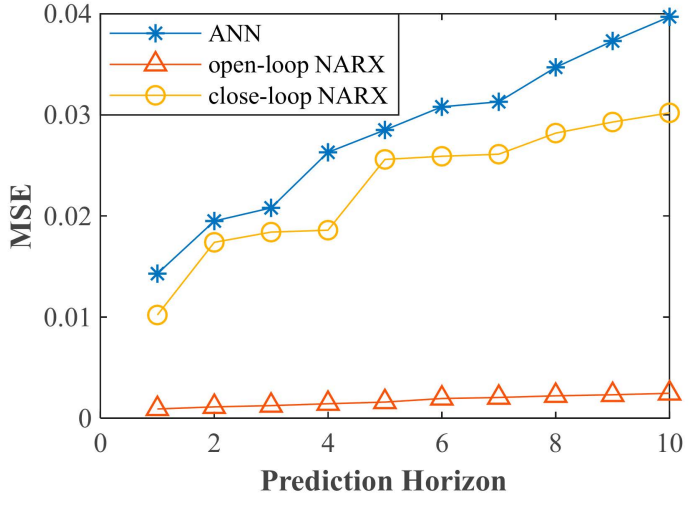

(a)

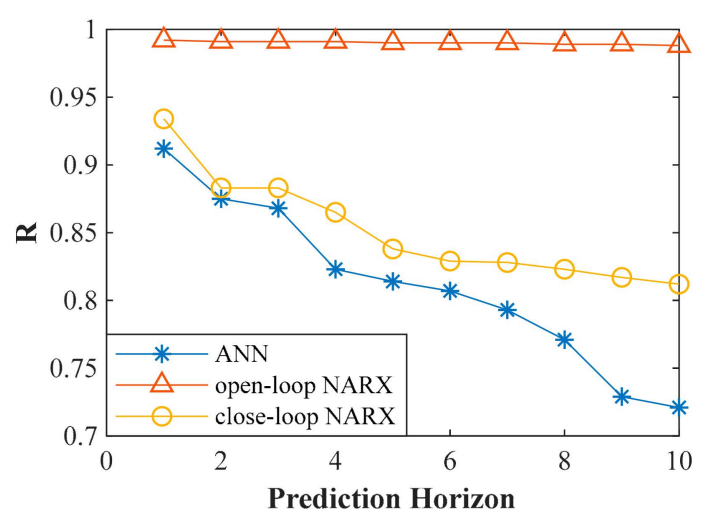

(b)

Figure 12. Performance of brake intensity predciton using Artificial Neural Networks (ANN) open-loop, and close-loop NARX networks. (a) Performance of the mean square error (MSE) with different prediction horizon; (b) Performance of the correlation coefficient (R) with different prediction horizon.

In general, the open-loop NARX network obtains better prediction performance in comparison to the close-loop NARX network and Artificial Neural Networks with Levenberg-Marquardt Backpropagation (LMBP) training algorithm. It can be found that the prediction performance was barely influenced, along with the increase of the prediction horizon for the open-loop NARX network. Figure 13 presents randomly selected brake intensity prediction results using the open-loop NARX network with 10 prediction horizon steps, which equal to $1 \mathrm{~s}$. It can be found that the predicted MCPs are quite close to real ones. Since the open-loop NARX network follows a direct prediction strategy, the output layer is composed of measurable data of the system's output (refer to MCP), 
which increases the complexity of the prediction model, but more accurate results are achieved [37]. On the other hand, the prediction performance of the close-loop NARX network and LMBP apparently deteriorated with the increase of the prediction horizon. The close-loop NARX network follows a recursive prediction strategy and estimated values as feedback are included in the output layer. In other words, MCP has to be intensively treated as an unmeasurable signal if the close-loop NARX network is adopted. The errors in the estimated MCPs are accumulated and transferred to the next prediction step as internal feedback, which inevitably deteriorates the accuracy of the brake intensity prediction performance.

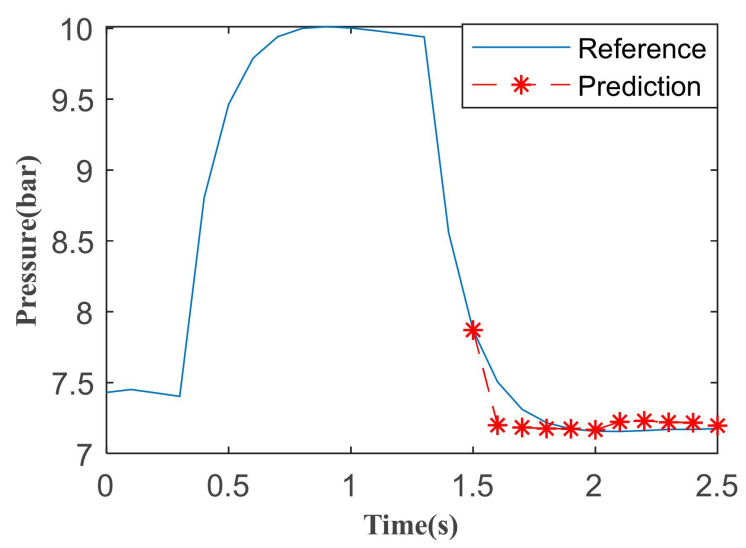

Figure 13. Brake intensity prediction results using the open-loop NARX network (memory order $=5$, neuron number $=20$ ) with 10 prediction horizon steps.

It is interesting that the prediction performance of the close-loop NARX network is still better than LMBP, although they employ the same training algorithm. Such differences become more apparent when the prediction horizon exceeds $7 \mathrm{~s}$. This behavior can be intuitively explained because the output memories of a NARX network emerge as jump-ahead connections in the time-unfolded network, which is often accompanied with backpropagation through the time algorithm. Such jump-ahead connections effectively shorten the paths for propagating gradient information, thus reducing the sensitivity of the network to long-term dependencies [38].

In conclusion, the open-loop NARX network becomes the first choice to predict brake intensity, since it can be directly indicated by MCP, which is a measurable signal. Nevertheless, with consideration of the system redundancy and fault tolerance of the braking system, the close-loop NARX network can be employed in case of possible CAN message dropout or MCP sensor failure.

\section{Conclusions}

In this paper, a novel composite machine learning approach is proposed for control-oriented prediction of driver brake intention and intensity. Fuzzy C-means clustering and random forest classification algorithms are coupled as a hybrid machine learning method to predict brake intention. A dynamic recurrent neural network, the NARX network with feedback and embedded memory, is applied to predict brake intensity. The conclusions are obtained as follows.

(1) Apart from the commonly used parameters in existing studies, various kinds of parameters that may potentially affect the performance of brake intention and intensity prediction are obtained from a FWD EV under real driving conditions. The rank importance of the selected predictors exhibit different trends or even negative trends when predicting brake intention and intensity, which indicates that ReliefF and RReliefF algorithms are qualified feature subset selection methods and can be applied in a prepossessing step before the model training.

(2) To deal with the randomness of the driver's brake intention, which is affected by driving style and traffic conditions, FCM, as a soft clustering algorithm, is effective in recognizing different brake intention levels. Compared with SVN, KNN, decision tree, and AdaBoost trees, RF yields the best 
performance, and the prediction accuracies with a prediction horizon of $0.5 \mathrm{~s}$ are $98.12 \%, 93.33 \%, 80.90 \%$, and $88.89 \%$ for slight, medium, intensive, and emergency braking, respectively. Further increases of the prediction time may deteriorate the prediction performance and be harmful to braking safety.

(3) In comparison to static neural networks, the open-loop NARX network is capable of learning long-term dependency and may become the first option for online recognition and prediction of brake intensity under real driving conditions if the master cylinder pressure is measurable. The prediction horizon can achieve at least $1 \mathrm{~s}$ prediction with extremely high accuracy. Meanwhile, with consideration of system redundancy and fault tolerance for an intelligent braking system, the close-loop NARX network can be considered in case of possible sensor failure or lost CAN message.

Author Contributions: Conceptualization, J.Z., J.S., L.H., Y.D., H.C. and W.Z.; Methodology, J.Z. and J.S.; Software, J.Z. and J.S.; Validation, J.Z. and J.S.; Formal Analysis, J.Z., J.S., L.H., Y.D., H.C. and W.Z.; Investigation, J.Z. and J.S.; Resources, J.Z.; Data Curation, J.Z., J.S.; Writing-Original Draft Preparation, J.Z., J.S.; Writing-Review \& Editing, J.Z., J.S.; Visualization, J.Z., J.S.; Project Administration, J.Z.; Funding Acquisition, J.Z.

Funding: This research was funded by the Fundamental Research Funds for the Central Universities grant number NS2016028.

Acknowledgments: The authors would like to thank the financial support from the Fundamental Research Funds for the Central Universities (Grant No. NS2016028).

Conflicts of Interest: The authors declare no conflict of interest.

\section{References}

1. Bärgman, J.; Boda, C.N.; Dozza, M. Counterfactual simulations applied to SHRP2 crashes: The effect of driver behavior models on safety benefit estimations of intelligent safety systems. Accid. Anal. Prev. 2017, 102, 165-180. [CrossRef]

2. Dumitru, A.I.; Girbacia, T.; Boboc, R.G.; Cristian, P.; Leonte, M. Effects of smartphone based advanced driver assistance system on distracted driving behavior: A simulator study. Comput. Hum. Behav. 2018, 83, 1-7. [CrossRef]

3. Guo, J.; Wang, J.; Cao, B. Regenerative braking strategy for electric vehicles. In Proceedings of the IEEE Intelligent Vehicles Symposium, Xi'an, China, 3-5 June 2009; pp. 864-868.

4. Song, C.; Li, S.; Zong, C. Fuzzy logic control method of EHB system based on braking intention pattern identification. In Proceedings of the IEEE International Conference on Automation and Logistics, Shenyang, China, 5-7 August 2009; pp. 1243-1247.

5. Li, W.; Du, H.; Li, W. Driver intention based coordinate control of regenerative and plugging braking for electric vehicles with in-wheel PMSMs. IET Intell. Transp. Syst. 2018, 12, 1300-1311. [CrossRef]

6. Zhou, Z.; Huang, M. Regenerative braking algorithm for the electric vehicle with a seamless two-speed transmission. J. Automob. Eng. 2019, 233, 905-916. [CrossRef]

7. Paul, D.; Velenis, E.; Cao, D. Optimal $\mu$-Estimation-Based Regenerative Braking Strategy for an AWD HEV. IEEE Trans. Transp. Electrif. 2016, 3, 249-258. [CrossRef]

8. Imamura, T.; Ogi, T.; Zhang, Z.; Tetsuo, M. Study of Induction and Estimation Method for Driver's Intention by Using a Driving Simulator. In Proceedings of the IEEE International Conference on Systems, Man, and Cybernetics, Manchester, UK, 13-16 October 2013; pp. 3322-3326.

9. Kost, F.; Schuh, J.; Koch-Dücker, H.J. Electronic Stability Program; ESP: Taunton, MA, USA, 2015.

10. Alonso, M.; Vega, M.H.; Martín, O. Driving simulation study for the analysis of distraction effects in longitudinal driving behaviour. Cogn. Technol. Work 2012, 14, 283-297. [CrossRef]

11. Fenzhu, J.I.; Farong, D.U.; Wenbo, Z. Electric vehicle energy economy based on braking intention identification. J. Beijing Univ. Aeronaut. Astronaut. 2016. [CrossRef]

12. Hernández, L.G.; Mozos, O.M.; Ferrández, J.M.; Antelis, J.M. EEG-based detection of braking intention under different car driving conditions. Front. Neuroinform. 2018, 12, 29. [CrossRef] [PubMed]

13. Haufe, S.; Kim, J.W.; Kim, I.H.; Sonnleitner, A.; Schrauf, M.; Curio, G.; Blankertz, B. Electrophysiology-based detection of emergency braking intention in real-world driving. J. Neural Eng. 2014, 11, 056011. [CrossRef]

14. Kim, I.H.; Bong, J.H.; Park, J.; Park, S. Prediction of driver's intention of lane change by augmenting sensor information using machine learning techniques. Sensors 2017, 17, 1350. [CrossRef] 
15. Li, F.; Wang, W.; Feng, G. Driving intention inference based on dynamic Bayesian networks. In Practical Applications of Intelligent Systems; Springer: Berlin/Heidelberg, Germany, 2014; pp. 1109-1119.

16. Zeng, X.; Wang, J. A stochastic driver pedal behavior model incorporating road information. IEEE Trans. Hum. -Mach. Syst. 2017, 47, 614-624. [CrossRef]

17. Zhao, X.; Xu, S.; Ye, Y.; Man, Y.; Wang, G. Composite braking AMT shift strategy for extended-range heavy commercial electric vehicle based on LHMM/ANFIS braking intention identification. Clust. Comput. 2018, 1-16. [CrossRef]

18. Xing, Y.; Lv, C.; Wang, H.; Xao, D. Recognizing driver braking intention with vehicle data using unsupervised learning methods. SAE 2017. [CrossRef]

19. Lv, C.; Xing, Y.; Lu, C.; Xao, D.; Gao, H.; Guo, H. Hybrid-learning-based classification and quantitative inference of driver braking intensity of an electrified vehicle. IEEE Trans. Veh. Technol. 2018, 67, 5718-5729. [CrossRef]

20. Zhao, X.; Wang, S.; Ma, J.; Gao, Y.; Man, Y. Identification of driver's braking intention based on a hybrid model of GHMM and GGAP-RBFNN. Neural Comput. Appl. 2018, 31, 1-14. [CrossRef]

21. Ding, N.; Yu, G.; Wang, W. Estimation of brake pressure and tyre-road friction during ABS activation. Int. J. Veh. Des. 2012, 58, 33-45. [CrossRef]

22. Ho, L.M.; Satzger, C.; de Castro, R. Fault-tolerant control of an electrohydraulic brake using virtual pressure sensor. In Proceedings of the International Conference on Robotics and Automation Sciences (ICRAS), Hong Kong, China, 26-29 August 2017; pp. 76-82.

23. Lv, C.; Xing, Y.; Zhang, J.; Na, X.; Li, Y.; Liu, T. Levenberg-Marquardt backpropagation training of multilayer neural networks for state estimation of a safety-critical cyber-physical system. IEEE Trans. Ind. Inform. 2017, 14, 3436-3446. [CrossRef]

24. Kim, J.W.; Kim, I.H.; Lee, S.W. Detection of braking intention during simulated driving based on EEG analysis: Online study. In Proceedings of the IEEE International Conference on Systems, Man, and Cybernetics, Kowloon, China, 9-12 October 2015; pp. 887-891.

25. Kim, I.H.; Kim, J.W.; Haufe, S.; Lee, S.W. Detection of braking intention in diverse situations during simulated driving based on EEG feature combination. J. Neural Eng. 2014, 12, 016001. [CrossRef]

26. Kumar, M.; Thenmozhi, M. Forecasting stock index movement: A comparison of support vector machines and random forest. In Proceedings of the Indian Institute of Capital Markets 9th Capital Markets Conference Paper, Mumbai, India, 24 January 2006.

27. Bezdek, J.C.; Ehrlich, R.; Full, W. FCM: The fuzzy c-means clustering algorithm. Comput. Geosci. 1984, 10, 191-203. [CrossRef]

28. Peker, M.; Arslan, A.; Sen, B.; Celebi, F.V.; But, A. A novel hybrid method for determining the depth of anesthesia level: Combining ReliefF feature selection and random forest algorithm (ReliefF+RF). In Proceedings of the International Symposium on Innovations in Intelligent SysTems and Applications (INISTA), Udaipur, Rajasthan, India, 18-20 September 2015; pp. 1-8.

29. Robnik-Šikonja, M.; Kononenko, I. Theoretical and empirical analysis of ReliefF and RReliefF. Mach. Learn. 2003, 53, 23-69. [CrossRef]

30. Liaw, A.; Wiener, M. Classification and regression by randomForest. R News 2002, 2, 18-22.

31. Menezes, J.M.P., Jr.; Barreto, G.A. Long-term time series prediction with the NARX network: An empirical evaluation. Neurocomputing 2008, 71, 3335-3343. [CrossRef]

32. Hochreiter, S.; Bengio, Y.; Frasconi, P. Gradient Flow in Recurrent Nets: The Difficulty of Learning Long-Term Dependencies; Wiley-IEEE Press: New York, NY, USA, 2001.

33. Siegelmann, H.T.; Horne, B.G.; Giles, C.L. Computational capabilities of recurrent NARX neural networks. Ieee Trans. Syst. ManCybern. 1997, 27, 208-215. [CrossRef]

34. Chetouani, Y. Using ARX and NARX approaches for modeling and prediction of the process behavior: Application to a reactor-exchanger. Asia-Pac. J. Chem. Eng. 2008, 3, 597-605. [CrossRef]

35. Sahoo, S.; Jha, M.K. Groundwater-level prediction using multiple linear regression and artificial neural network techniques: A comparative assessment. Hydrogeol. J. 2013, 21, 1865-1887. [CrossRef]

36. Xie, H.; Tang, H.; Liao, Y.H. Time series prediction based on NARX neural networks: An advanced approach. In Proceedings of the International conference on machine learning and cybernetics, Hebei, China, 12-15 July 2009; pp. 1275-1279. 
37. Capraro, F.; Patino, D.; Tosetti, S.; Schugurensky, C. Neural network-based irrigation control for precision agriculture. In Proceedings of the IEEE International Conference on Networking, Sensing and Control, Sanya, China, 6-8 April 2008; pp. 357-362.

38. Giles, C.L.; Lin, T.; Horne, B.G. Remembering the past: The role of embedded memory in recurrent neural network architectures. In Proceedings of the Neural Networks for Signal Processing VII. Proceedings of the 1997 IEEE Signal Processing Society Workshop, Amelia Island, FL, USA, 24-26 September 1997; pp. 34-43.

(C) 2019 by the authors. Licensee MDPI, Basel, Switzerland. This article is an open access article distributed under the terms and conditions of the Creative Commons Attribution (CC BY) license (http://creativecommons.org/licenses/by/4.0/). 